\title{
CASCADES OF PERIOD-DOUBLING BIFURCATIONS: A PREREQUISITE FOR HORSESHOES ${ }^{1}$
}

\author{
BY JAMES A. YORKE AND KATHLEEN T. ALLIGOOD
}

Since Smale [7] described the complicated dynamical behavior of horseshoe maps, many dynamical systems depending on a parameter have been shown to develop horseshoes. In the horseshoe, there are $2^{k}$ fixed points for the $k$ th iterate of the map. Numerical studies of parametrized maps and the investigations of Newhouse [5] and Robinson [6] indicate there is a rich structure of attractors for parameters preceding the existence of the horseshoe. Once the horseshoe is formed, however, all periodic points are unstable and almost any trajectory starting in the horseshoe eventually leaves it. Assuming that cross-sectional areas are contracting, we prove that infinitely many cascades of period doublings must occur in the process of forming a horseshoe. Each such cascade need not evolve regularly or monotonically, but it must contain attracting periodic points of all the periods $k, 2 k, 4 k, 8 k, \ldots$, for some $k$. For the area preserving case with $n=2$, we have an analogous result, where elliptic periodic points replace attracting periodic points.

We would like to thank L. Tedeschini-Lalli and S. Pelikan for their helpful suggestions.

First, we introduce notation and give hypotheses for the formation of horseshoes. Let

$$
\begin{aligned}
& C=\left\{\left(x_{1}, \ldots, x_{n}\right) \in R^{n}: x_{1}^{2}+\cdots+x_{n-1}^{2} \leq 1 \text { and } 0 \leq x_{n} \leq 1\right\}, \\
& E=\left\{\left(x_{1}, \ldots, x_{n}\right) \in C: x_{1}^{2}+\cdots+x_{n-1}^{2}=1\right\}, \\
& T=\left\{\left(x_{1}, \ldots, x_{n}\right) \in C: x_{n}=1\right\}, \text { and } \\
& B=\left\{\left(x_{1}, \ldots, x_{n}\right) \in C: x_{n}=0\right\} .
\end{aligned}
$$

Let $f: C \times[0,1] \rightarrow R^{n}$ be a $C^{1}$ map such that $f(C \times\{0\})$ is disjoint from $C$, and $f(\cdot, 1)$ is a horseshoe map, in the sense of Smale [7]. Specifically, writing $C_{\lambda}$ for $f(C \times\{\lambda\})$ and using corresponding notation for $E, T$, and $B$, we assume:

(H1) There is a $\theta<1$ such that if $f^{k}(p, \lambda)=p$ for some $\lambda \in[0,1], k \geq 1$, and $p \in C$, and if $\mu_{1}, \ldots, \mu_{n}$ are the eigenvalues of $D_{p} f^{k}(p, \lambda)$, then $\left|\mu_{i} \mu_{j}\right|<\theta$ for $i \neq j, 1 \leq i, j \leq n$;

(H2) $C_{0} \cap C$ is empty;

(H3) $T_{\lambda} \cap C$ and $B_{\lambda} \cap C$ are empty, for all $\lambda, 0 \leq \lambda \leq 1$;

(H4) $C_{\lambda} \cap E$ is empty, for all $\lambda, 0 \leq \lambda \leq 1$; and

Received by the editors April 6, 1983.

1980 Mathematics Subject Classification. Primary 58F12, 58F13, 58F14, 58F20.

${ }^{1}$ Research supported by the National Science Foundation (Alligood and Yorke) and by the Air Force Office of Scientific Research (Yorke). 
(H5) If $f^{k}(p, 1)=p$, for some $k \geq 1, p \in C$, then $D_{p} f^{k}(p, 1)$ has an eigenvalue $\mu$ such that $|\mu|>1$. (For each $k$, there are $2^{k}$ such points.)

In addition, let $P=\left\{(p, \lambda) \in C \times[0,1]: f^{k}(p, \lambda)=p\right.$, for some $\left.k\right\}$; and for a point $(p, \lambda) \in P$, let $\Lambda(p, \lambda)=\left\{\mu: \mu\right.$ is an eigenvalue of $D_{p} f^{k}(p, \lambda)$, where $k$ is the minimum period of $(p, \lambda)\}$.

TheOREM 1. Let $f$ satisfy (H1)-(H5). For $(p, 1) \in P$, the component of $P$ containing $(p, 1)$ has attracting periodic points of minimum period $2^{n} k$, for each $n \geq 0$.

The main ideas in the proof of this theorem are outlined in the lemmas and discussion which follow. First, we form the orbit space $O(f)$ of equivalence classes of periodic points of $f$ : identify points $(p, \lambda)$ and $(q, \lambda)$ in $P$ if $f^{m}(p, \lambda)=$ $q$, for some $m$. We write $O(f, k)$ for orbits of $f$ of minimum period $k$. Hence, $O(f)=\bigcup_{k \in N} O(f, k)$. For ease of notation, we write $(p, \lambda) \in O(f)$ for any representative $(p, \lambda)$ of an orbit. By (H1), each orbit in $O(f)$ is in one of the following five disjoint subsets, classified according to the location of the $\mu$ in $\Lambda(p, \lambda)$ :

(1) the set $A$ of attracting orbits $(|\mu|<1$, for all $\mu$ );

(2) the set $M$ of Mobius orbits $(\mu<-1$, for some $\mu)$;

(3) the set $S$ of saddle orbits $(\mu>1$, for some $\mu)$;

(4) $B_{+}=\{(p, \lambda) \in O(f): 1 \in \Lambda(p, \lambda)\}$; and

(5) $B_{-}=\{(p, \lambda) \in O(f):-1 \in \Lambda(p, \lambda)\}$.

Now we analyze the structure of path components of orbits for a particular generic class $G$ of functions in $C^{1}\left(C \times I, R^{n}\right)$. The set $G$ is discussed in detail in [1 and 2]. Here we mention that the types of orbits in $B_{+}$and $B_{-}$are restricted for $g \in G$ :

(1) Orbits in $B_{+} \cap \mathcal{O}(g)$ are "saddle-node" bifurcation orbits from which two paths of non-Mobius orbits emanate: one path is a subset of $S$ and one is a subset of $A$. Near the saddle-node, orbits on both paths have the same minimum period as the bifurcation orbit.

(2) Orbits in $B_{-} \cap \mathcal{O}(g)$ are "period-doubling" bifurcation orbits from which 3 paths of orbits - two non-Mobius and one Mobius emanate. Near the bifurcation orbit, each of the non-Mobius paths is a subset either of $A$ or of $S$ depending on the direction of the path in $\lambda$. (See index discussion below.) Orbits on one of the non-Mobius branches have twice the minimum period of the bifurcation orbit, while orbits on the other two branches have the same minimum period. In addition, an analysis of the eigenvalues near the bifurcation orbit (under (H1)) shows that the low period non-Mobius orbits must be attractors (i.e., saddle orbits cannot "period-double").

From the description of generic orbits, we obtain the following lemma:

LemMA 1 [2]. Let $g \in G$. Each non-Mobius orbit in $O(g)$ is contained in a (topological) 1-manifold $\Gamma$ of non-Mobius orbits of $O(g)$.

The critical step in proving Theorem 1 is the use of the orbit index, as developed for generic maps in [3] and [4], to orient the 1-manifold $\Gamma$. If $(p, \lambda)$ is not a bifurcation orbit, then its index, denoted $\varphi(p)$, is +1 for attractors, 0 for Mobius orbits, and -1 for saddles. (See [4] for the precise definition.) 
If the periods of orbits on $\Gamma$ are bounded, then $\Gamma_{\lambda_{0}}=\left\{(p, \lambda) \in \Gamma: \lambda=\lambda_{0}\right\}$ is finite, and $\sum \varphi(p)$, for $\left(p, \lambda_{0}\right) \in \Gamma_{\lambda_{0}}$, is invariant under changes in $\lambda$. The invariance property of $\varphi$ allows us to choose a preferred direction on $\Gamma$ in the following manner:

(1) if $\varphi(p)=+1$, follow $\Gamma$ through increasing $\lambda$ values;

(2) if $\varphi(p)=-1$, follow $\Gamma$ through decreasing $\lambda$ values.

Hence $\Gamma$ changes direction (from $\lambda$ increasing to $\lambda$ decreasing, or vice versa) at orbits in $B_{+}$. It may or may not change direction at orbits in $B_{-}$, depending on the types of orbits of the two non-Mobius branches.

Now let $(q, 1)$ be a saddle orbit in $O(g, k)$, and let $\Gamma$ be the oriented 1manifold of non-Mobius orbits which begins at $(q, 1)$. We follow $\Gamma$ through decreasing $\lambda$ values from $(q, 1)$, as its orientation requires. Since there are no (periodic) orbits in $C_{0}$, either $\Gamma$ must change direction or all nonbifurcation orbits on $\Gamma$ are saddles, and the periods of these orbits are unbounded. Since saddle orbits cannot "period-double", the second case is impossible. Hence $\Gamma$ contains attractors. Furthermore, $\Gamma$ cannot reintersect $C_{1}$ since the direction of approach (through increasing $\lambda$ ) would imply that $\Gamma \cap C_{1}$ contains either an attractor or a bifurcation orbit, both of which are impossible. Thus $\Gamma-\{(q, 1)\}$ is contained in $C \times(0,1)$. The global continuation results of [2] and [4] give the following lemma:

LeMMA 2. Let $g \in G$, and let $(q, 1) \in \mathcal{O}(g, k)$ be a saddle orbit on a path $\Gamma$ of non-Mobius orbits (as in Lemma 1). Then the minimum periods of orbits in $\Gamma$ are unbounded. Furthermore, there is an attractor of minimum period $2^{n} k$ on $\Gamma$, for each $n \geq 0$.

The statement about the existence of attractors follows from the fact that saddle orbits cannot "period-double". An analysis of generic bifurcations provides an analogous result for $(q, 1) \in M$, if we follow a path of Mobius orbits from $(q, 1)$ through decreasing $\lambda$ to a period-doubling bifurcation. Finally, we take limits of the generic paths described in Lemma 2 and use the "virtual period" techniques developed in [1 and 2] to obtain the result in the general case (Theorem 1).

The "virtual period" techniques also give information as to the number of attractors of each minimum period in the limit case. We define a cascade from period $k$ to be a sequence $\left\{K_{n}\right\}_{n \in N}$ of (nonempty) components of $A$ such that $K_{0}$ is a subset of $O(f, k)$, and if $(p, \lambda) \in K_{n}$, then the minimum period of $(p, \lambda)$ is $2^{n} k$. Furthermore, we require that $\bigcup_{n \in N} K_{n}$ is contained in one component of $P$.

THEOREM 2. Let $f$ satisfy (H1)-(H5), and let $N$ be the number of saddle orbits of minimum period $k$ in $C_{1}$. Then there are at least $N$ disjoint cascades from period $k$ in $O(f)$.

\section{REFERENCES}

1. K. T. Alligood, J. Mallet-Paret and J. A. Yorke, An index for the global continuation of relatively isolated sets of periodic orbits, Proc. Dynamics Sympos. IMPA, Rio de Janeiro, 1981 (to appear). 
2. K. T. Alligood and J. A. Yorke, Families of periodic orbits: virtual periods and global continuability, J. Differential Equations (to appear).

3. S. N. Chow, J. Mallet-Paret and J. Yorke, A bifurcation invariant: degenerate orbits treated as clusters of simple orbits, Proc. Dynamics Sympos. IMPA, Rio de Janeiro, 1981 (to appear).

4. J. Mallet-Paret and J. Yorke, Snakes: oriented families of periodic orbits, their sources, sinks and continuation, J. Differential Equations 43 (1982), 419-450.

5. S. Newhouse, Lectures on dynamical systems, Dynamical Systems, Progress in Mathematics, Vol. 8, Birkhäuser, Boston, 1980.

6. C. Robinson, Bifurcation to infinitely many sinks, preprint.

7. S. Smale, Differentiable dynamical systems, Bull. Amer. Math. Soc. 73 (1967), 747-817.

Department of Mathematics and Institute for Physical Science and Technology, University of Maryland, College Park, Maryland 20742

Department of Mathematics, College of Charleston, Charleston, South Carolina 29424

Current address (K. T. Alligood): Department of Mathematics, Michigan State University, East Lansing, Michigan 48824 